\title{
Is Moderate Red Wine Consumption Safe in Inactive Inflammatory Bowel Disease?
}

\author{
Garth R. Swanson Vanessa Tieu Maliha Shaikh Chris Forsyth Ali Keshavarzian \\ Department Digestive Diseases, Rush University Medical Center, Chicago, III., USA
}

\section{Key Words}

Crohn's disease • Ulcerative colitis · Alcohol consumption

\begin{abstract}
Background: Alcohol consumption is a potential trigger for inflammatory bowel disease (IBD) flare because of alcoholinduced oxidative stress and its deleterious effects on gut barrier function. Additionally, we have recently shown that alcohol consumption is associated with more symptoms in IBD. However, it is not known whether moderate daily alcohol consumption can modify IBD disease activity. To test what effects alcohol may have on patients with IBD, we evaluated the effect of moderate daily red wine for 1 week on two factors associated with recurrent IBD disease activity: intestinal permeability and stool calprotectin. Methods: To assess the effects of moderate daily alcohol consumption on intestinal permeability and inflammation, we recruited 21 patients: 8 with inactive ulcerative colitis (UC), 6 with inactive Crohn's disease (CD), and 7 healthy controls. All participants with IBD completed a validated questionnaire on disease activity (Crohn's disease activity index or ulcerative colitis clinical activity index), to confirm they had inactive disease. All subjects then underwent a baseline assessment that included a blood draw, urine collection after sugar challenge, and
\end{abstract}

stool collection. Subjects then consumed 1-3 glasses of red wine a day for 1 week (approx. $0.4 \mathrm{~g} \mathrm{EtOH} / \mathrm{kg}$ ), and repeated the three measures. Results: No subjects flared during the study. Moderate alcohol consumption did not significantly change either clinical disease activity scores or C-reactive protein. In contrast to healthy subjects, daily consumption of red wine significantly (1) decreased stool calprotectin in IBD subjects from baseline $(p=0.001)$ and $(2)$ increased intestinal permeability as measured by urinary lactulose/mannitol excretion (marker of small bowel permeability) in $C D(p=0.028)$ or urinary sucralose secretion (marker of large bowel permeability) in UC ( $p=0.012)$. Conclusions: One week of moderate consumption of red wine in inactive IBD was associated with a significant decrease in stool calprotectin and a significant increase in intestinal permeability. Our data suggests that patients with inactive IBD who drink red wine daily may be at an increased long-term risk for disease relapse.

Copyright $\odot 2011$ S. Karger AG, Basel

\section{Introduction}

Inflammatory bowel disease (IBD) has a waxing and waning course, with periods of asymptomatic disease inactivity (remission) interrupted by episodes of symptom-

\section{KARGER}

(c) 2011 S. Karger AG, Basel

Fax +41613061234

E-Mail karger@karger.ch

www.karger.com
Accessible online at: www.karger.com/dig
Garth R. Swanson, MD, MS

Department Digestive Diseases, Rush University Medical Center

1725 West Harrison Street, Suite 207

Chicago, IL 60612-3824 (USA)

Tel. +1 312563 3871, E-Mail garth_r_swanson@rush.edu 
atic disease activity (flare). Although the causes for disease flare have not been fully established, several environmental factors have been suggested as potential triggers [1]. Of course, identifying these factors is important as they are potentially modifiable elements that could limit the use of steroids and decrease hospitalizations. The best studied environmental factor in IBD is cigarette smoking. Cigarette smoking has been shown to increase the need for steroids and frequency of surgery in Crohn's disease (CD) [2], and quitting smoking improves the course of disease [3]. On the other hand, in ulcerative colitis (UC), smoking reduces the risk of colectomy [4], decreases the extent of disease activity in the colon, and delays the age of presentation [5].

Another commonly used product with potential impact in inflammatory processes of the gut is alcohol. Indeed, alcohol consumption can modulate several pathways that are involved in pathogenesis of tissue inflammation and tissue injury in IBD. For example, alcohol affects immune states and immune response in both humans and rodents [6, 7]. Moreover, alcohol consumption can disrupt intestinal barrier function $[8,9]$ and affect microbiota composition [10]. Thus, alcohol consumption can potentially impact IBD disease course. However, the effect of alcohol in IBD has been largely unstudied. One prospective cohort study on the effects of diet in UC found that patients with diets high in either alcohol or meat were at higher risk for flare over the next year [11]. Another study in CD found no difference in alcohol absorption or abdominal pain from five different alcoholic drinks [12], and concluded that it is likely the high sugar content that is responsible for reported symptoms. In addition, we recently reported on a cohort study using a survey analysis of alcohol consumption in inactive IBD, and found that patients with IBD do drink alcohol in a similar pattern to the general population, but were also more likely to report that alcohol worsens their gastrointestinal (GI) symptoms than patients with irritable bowel syndrome [13].

However, there is no study that has directly assessed the effect of moderate social drinking on markers of disease activity in IBD. Accordingly, the aims of the current study were to determine the effects of daily red wine consumption in inactive IBD on (1) clinical disease activity and (2) noninvasive markers of disease activity: C-reactive protein (CRP), stool calprotectin, and intestinal permeability. We chose to study intestinal permeability and stool calprotectin because abnormal permeability [14-16] and elevated stool calprotectin [17] have been shown to be associated with increased risk of future disease flare.

\section{Patients and Methods}

\section{Study Design}

This was a prospective cohort study done in patients with IBD. Patients were recruited from a GI clinic in a metropolitan university practice in Chicago, Ill., USA (Rush University Gastroenterology) from January 2007 until December 2009. During this period, patients who were in clinical remission with an established diagnosis of CD or UC and health subjects with no gastrointestinal diagnosis were invited to participate in the study. All participants were social drinkers and were willing to drink red wine daily for 7 days. After signing an informed consent, all patients were given a set of questionnaires to complete. The questionnaire packet included a demographic data form, a validated questionnaire on disease activity [Crohn's disease activity index (CDAI) or ulcerative colitis clinical activity index (UCAI), respectively], and a questionnaire quantifying the amount of alcohol subjects usually consumed based on established criteria by the National Institute on Alcohol Abuse and Alcoholism (NIAAA). All questionnaire packets were labeled by a sequential patient number to maintain patient confidentiality, and served as the patient identifier for the remainder of the study.

Once subjects were enrolled, participants were asked not to drink for 1 week prior to the baseline assessment for the study. At the baseline assessment, subjects underwent a blood draw, stool collection, and urine collection after ingestion of the sugar mixture. Each patient then consumed 1-3 glasses of red wine per day (approx. $0.4 \mathrm{~g}$ of alcohol/ $\mathrm{kg}$ of body weight) for 1 week. The volume of a standard drink of red wine with $12 \mathrm{~g}$ of alcohol was explained to each subject prior to beginning the study. The red wine was supplied to each subject, and was the same year and vintage - a Chilean Cabernet Sauvignon 2003. All subjects were told not to drink any other alcohol during the week of the study. After completing 7 days of alcohol consumption, subjects then returned for repeat measures including a blood draw, stool collection, and urine collection after ingestion of the sugar mixture.

The study was approved by Rush University Investigational Review Board (Chicago, Ill., USA).

\section{Inclusion Criteria and Exclusion Criteria}

All IBD patients included in the study had biopsy-proven CD or UC. IBD patients were eligible to be included in the study if the clinician who saw them at the time of inclusion determined that they currently had inactive disease. The status of disease for IBD patients was then confirmed using IBD clinical indices: CDAI $<150$ or UCAI $<4$ for inactive disease. Patients were excluded from the study if they reported active disease in the 3 months prior to the enrollment. Patients were also excluded from the trial if they were on biologic medications (infliximab, adalimumab, etc.) for maintenance, as the periodic dosing of these medications could be a confounder in this 1-week trial. All participants included in the study had been on a stable dose of their IBD medications for at least 3 months and were not taking NSAIDs for at least 4 weeks prior to beginning the study. Patients were included as healthy controls (HC) if they had no established diagnosis of GI disease and were required to complete a questionnaire marking 'no' on a list of common GI diseases and symptoms. Finally, subjects with any history of alcohol or drug abuse, either IBD patients or controls, were excluded from participation in the trial. 
Questionnaires

Demographic and Medical History Data Form. The demographic variables we collected included age, race, marital status, education, occupation, and alcohol consumption. In addition, patients were asked to indicate different factors regarding the history of IBD, i.e. length of duration of diagnosis, recent disease activity, medication history, and family history of GI medical conditions.

IBD Disease Activity Indices. We used two validated questionnaires to access disease activity: the CDAI and UCAI, both of which have been well described previously [18]. These clinical questionnaires have IBD patients rate their current symptoms based on clinical questions, i.e. stool frequency, abdominal pain, general well-being, etc., and were completed by the primary investigator (G.R.S.), who saw them at each visit.

\section{Measures}

Stool Calprotectin. Stool samples were collected at baseline and after alcohol consumption by the patients. All stool samples were sent to Genova Diagnostics (Ashville, N.C., USA) where fecal calprotectin was measured by quantitative enzyme-linked immunosorbent assay (ELISA) or PhiCal test immediately after collection.

Intestinal Permeability Measurement. Intestinal permeability was assessed using a poorly absorbed sugar test that we have published previously [19]. After an 8-hour fast, each subject ingested $150 \mathrm{ml}$ of liquid containing $10 \mathrm{~g}$ of lactulose, $5 \mathrm{~g}$ of mannitol, and $40 \mathrm{~g}$ of sucrose with 4 capsules each containing $500 \mathrm{mg}$ of sucralose. Before ingestion of the test sugar, each subject was asked to empty his bladder completely. Thereafter, all urine was collected for $24 \mathrm{~h}$. Urine volumes were recorded and aliquots of urine were stored at $-80^{\circ} \mathrm{C}$ until analysis. Measurement of urinary sugars was done by gas chromatography using a modified method we previously published. Urinary sugar excretion was calculated and presented as a percentage of oral intake. We [20] and others have shown that the lactulose/mannitol ratio $(\mathrm{L} / \mathrm{M})$ represents a reliable marker of small bowel permeability. Sucralose excretion represents whole gut permeability including the colon because, unlike lactulose or mannitol, sucralose is not fermented by colonic bacteria and is thus available for absorption in the colon [21].

Blood Collection. The blood draw included CRP, complete blood count with differential, and comprehensive metabolic panel. All studies were performed at the Rush University outpatient laboratories. CRP is the most commonly used noninvasive inflammatory marker in IBD, and it has been used to predict placebo response rates in clinical trials [22].

\section{Statistical Analysis}

We used the Wilcoxon signed-rank test to compare differences between the repeated measures in each group. We used a $p$ value of 0.05 as significant for all tests, and data was analyzed using SPSS version 12.0.1 (SPSS Inc., Chicago, Ill., USA).

\section{Results}

\section{Patient Characteristics}

A total of 23 subjects completed the study. Two patients were excluded from the trial as one was unable to drink the wine, and another did not drink any sugars prior to
Table 1. Patient characteristics by group

\begin{tabular}{|c|c|c|c|}
\hline & $\begin{array}{l}\text { CD } \\
(n=6)\end{array}$ & $\begin{array}{l}\text { UC } \\
(n=8)\end{array}$ & $\begin{array}{l}\text { Controls } \\
(n=7)\end{array}$ \\
\hline Median age, years & 31 & 45 & 24 \\
\hline Male sex & $4(66)$ & $4(50)$ & $3(43)$ \\
\hline Median disease duration, years & 7.6 & 15 & $\mathrm{n} / \mathrm{a}$ \\
\hline \multicolumn{4}{|l|}{ Median CDAI or UCAI } \\
\hline Before wine consumption & 11.5 & 2.0 & $\mathrm{n} / \mathrm{a}$ \\
\hline After wine consumption & 4.0 & 1.0 & $\mathrm{n} / \mathrm{a}$ \\
\hline Median CRP & $<5.0$ & $<5.0$ & $<5.0$ \\
\hline Baseline median calprotectin & 75 & 745 & 11 \\
\hline \multicolumn{4}{|l|}{ Baseline alcohol consumption } \\
\hline$\%$ light & $2(33)$ & $7(88)$ & $3(43)$ \\
\hline \multicolumn{4}{|l|}{ GI area involved } \\
\hline Colon only & $2(33)$ & $8(100)$ & $\mathrm{n} / \mathrm{a}$ \\
\hline Ileum only & $2(33)$ & 0 & $\mathrm{n} / \mathrm{a}$ \\
\hline Colon and ileum & $2(33)$ & 0 & $\mathrm{n} / \mathrm{a}$ \\
\hline \multicolumn{4}{|l|}{ Medications } \\
\hline None & $1(17)$ & $1(13)$ & $7(100)$ \\
\hline $5 \mathrm{ASA}$ & $4(66)$ & $3(38)$ & 0 \\
\hline 6MP, AZA, MTX & $1(17)$ & $4(50)$ & 0 \\
\hline
\end{tabular}

Figures in parentheses are percentages.

the urine collection and did not collect stool. The remaining 21 subjects completed the trial: 8 with UC, 6 with CD, and $7 \mathrm{HC}$. Subject characteristics are listed in table 1 . The median age was 45 years in UC subjects, 31 in CD, and 24 in the HC. IBD subjects were predominately male (88\%), while the $\mathrm{HC}$ were predominately female (56\%). Although the IBD subjects were older and there were more men enrolled, the median age and gender were not significantly different from the HC ( $\mathrm{p}=0.158$ and 0.58 , respectively). The majority of IBD subjects had colonic involvement (12; $75 \%)$ and $5(36 \%)$ of the IBD subjects were on an immunomodulator: azathioprine or methotrexate. There was no significant difference between $\mathrm{CD}$ or UC on immunomodulators. The amount of baseline alcohol consumption prior to enrollment in the study was recorded by a validated questionnaire according to NIAAA criteria and was slightly more in patients with CD than UC (33 to 88\% light drinkers). Light drinkers are defined as having less than 3 drinks a week, with the remainder of patients either classified as moderate or heavy drinkers.

\section{Clinical Disease Activity and CRP}

No patients flared or experienced a worsening of their symptoms or diarrhea during the trial. Baseline levels of clinical activity indices, the UCAI and CDAI, were 11.5 


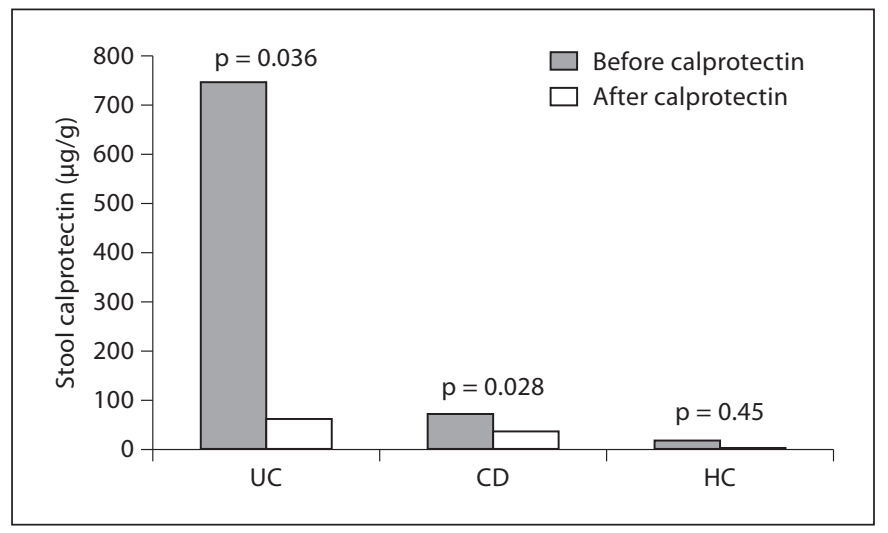

Fig. 1. Median stool calprotectin before and after moderate red wine consumption.

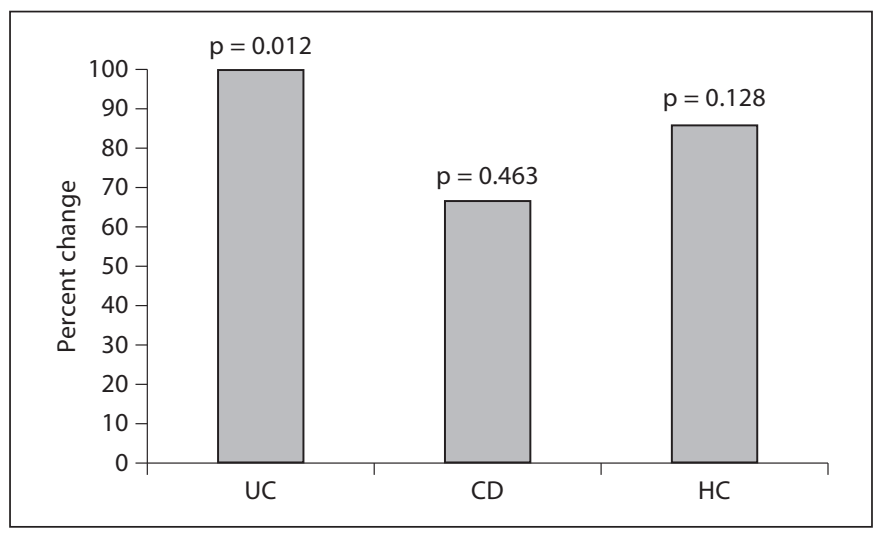

Fig. 3. Increased permeability by urinary sucralose excretion after moderate wine consumption.

and 4.0, respectively. There was no significant difference at a baseline between the two groups, or change in either clinical activity index after wine consumption. The median CRP in both groups, IBD and HC, was less than 5.0, which was the lower limit of detection at our lab. There was no change in CRP level associated with moderate wine consumption.

\section{Stool Calprotectin}

Although all patients were clinically inactive and had low disease activity indices in order to participate in the trial, the baseline stool calprotectin levels varied significantly and 7 of 8 patients with UC (88\%) and 4 of 6 patients with CD (67\%) had elevated levels at baseline (ta-

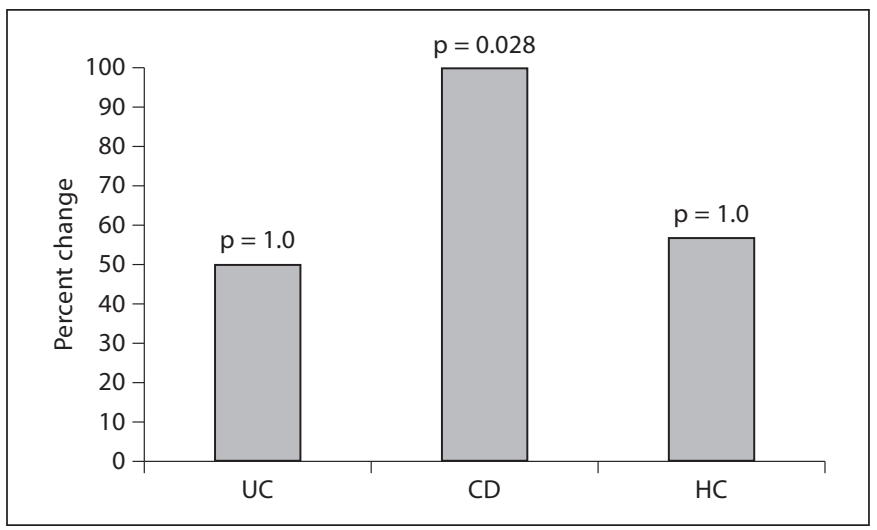

Fig. 2. Increased permeability by urinary L/M ratio after moderate wine consumption.

ble 1). As expected, stool calprotectin was low in healthy subjects. Alcohol had no significant effects on stool calprotectin ( $p=0.45$; fig. 1 ). Stool calprotectin levels ranged from low normal to high in IBD patients in spite of the fact that all were clinically inactive with a low disease activity index (fig. 1). In contrast to healthy subjects, daily consumption of wine significantly affected stool calprotectin in IBD patients. After 1 week of daily consumption of red wine, there was a significant reduction in stool calprotectin in IBD subjects compared to baseline ( $\mathrm{p}=$ $0.001)$.

\section{Intestinal Permeability}

Values of the baseline urinary L/M ratio (marker of small bowel permeability) were not significantly different in inactive IBD patients $(\mathrm{UC}=0.0853, \mathrm{CD}=0.0847)$ from HC (0.0935). Similarly, urinary sucralose (marker of total gut permeability) was similar among three groups ( $\mathrm{UC}=$ $1.538, \mathrm{CD}=1.252$, and $\mathrm{HC}=0.779$ ). The effects of daily wine consumption on intestinal permeability are shown in figures 2 and 3 . The urinary $\mathrm{L} / \mathrm{M}$ ratio and urinary sucralose values were not significantly affected by 1 week of daily consumption of wine in healthy subjects. In contrast, daily wine consumption significantly affected intestinal permeability in IBD patients. The deleterious effects of wine consumption on intestinal permeability were selective and the increase in intestinal permeability correlated with known location of disease. There was a significant increase in urinary $\mathrm{L} / \mathrm{M}$ excretion in $\mathrm{CD}$ subjects $(\mathrm{p}=0.028)$, but not in patients with UC. The urinary sucralose excretion was significantly increased in UC $(\mathrm{p}=0.012)$, but not in CD. 


\section{Discussion}

One of the most important long-term therapeutic goals in IBD is to prevent disease flare and maintain remission. This goal can ideally be achieved if the triggers for IBD flare are identified and thus avoided. Although several environmental factors have been proposed as triggers for IBD flare, the role of environmental factors as a cause for triggering a disease flare in IBD, unfortunately, is still controversial and unproven - for most factors. The best studied factor that can clearly impact disease activity has been cigarette smoking, while studies in diet [23], stress [24], and sleep [25] have been intriguing but inconclusive. This study attempts to quantify the effect of another common environmental factor - red wine.

Alcohol consumption is an important environmental exposure in IBD. Indeed, previous studies have already demonstrated that alcohol is a common exposure in patients with IBD, and it is consumed in proportion to the general population [13]. There is no doubt that hazardous/harmful alcohol use (defined as consumption of greater than $40 \mathrm{~g}$ of alcohol per day for men and $20 \mathrm{~g}$ for women) [26] has numerous deleterious health effects on other organ systems like the liver, intestine, and immune system and incurs annual health costs of USD 100 billion in the United States [27]. It remains to be determined whether more moderate social drinking also has deleterious health effects in those with chronic inflammatory disorders like IBD. This is an important question to know because patients with IBD commonly ask their gastroenterologist whether alcohol will affect their disease course or risk for disease flare.

Several physiological effects of alcohol raise a possibility that even moderate and social alcohol consumption might affect intestine and immune response as such to impact IBD disease course. For example, alcohol can decrease dendritic cell function and increase the inhibitory cytokine IL-10 [28], thus potentially inhibiting the inflammatory pathway and preventing IBD flare-up. In contrast, alcohol can also increase immune activity by increasing intestinal permeability and causing subsequent endotoxemia [29]. Patients with IBD have increased levels of endotoxins, which may correlate with disease activity [30]. Patients with IBD have exaggerated immune response to endotoxin and epithelial dysfunction, which can cause alterations in the innate and adaptive immune system [31]. Thus, alcohol-induced gut leakiness can further increase endotoxemia and potentially trigger IBD flare-up. However, there has been no study to date to determine which of these two diverging effects of alcohol is operational in IBD. This type of study is essential in order for clinicians to correctly advise patients with IBD in regards to consumption of alcoholic beverages.

Accordingly, we conducted our study to determine the effects of short-term moderate social drinking on intestinal inflammation and intestinal permeability in patients with inactive IBD. We prospectively followed patients with inactive IBD during moderate red wine consumption. There was no change in clinical disease activity indices or CRP levels in subjects with inactive IBD after 1 week of the daily consumption of 2-3 glasses of red wine (24-36 g of alcohol). This was expected as all subjects were inactive by definition at the start of the study, and the follow-up time for moderate alcohol consumption was only 7 days. Seven days was chosen as a duration for the study to limit the dropout rate, and this time period also represents about two half-lives for the intestinal mucosa, thus any acute change in the intestinal mucosal immune activity or function might be appreciated.

In contrast to clinical indices, consumption of red wine significantly impacted two subclinical markers of disease activity: stool calprotectin and intestinal permeability. Stool calprotectin has been shown to be associated with recurrent disease in IBD [17]. Interestingly, this study showed a marked decrease in stool calprotectin after 1 week of moderate red wine consumption. The effect of wine consumption on stool calprotectin was more marked in patients with UC as stool calprotectin has been shown to be a better marker of disease activity in UC than $\mathrm{CD}$ [32]. However, it should be noted that our inactive UC group had elevated baseline values for calprotectin, but normal clinical indices. The reason for this reduction in stool calprotectin is likely due to the effects of alcohol on inhibiting the systemic immune system and neutrophil migration [6]. It must also be noted that the lowering of stool calprotectin levels by red wine consumption in IBD patients could be due to other components in the wine, such as the antioxidant resveratrol, which is a known antioxidant with anti-inflammatory effects [33].

Resveratrol is a nonflavonoid polyphenol rich in red wine and grapes which has beneficial health effects from its antioxidant, anticancer, and anti-inflammatory properties. Resveratrol is known to enhance the innate immunity through TLR4 [34] and decrease endotoxin-induced expression of inflammatory cytokines. Whether the alcohol or another substance in the red wine was responsible for the decrease in stool calprotectin could not be assessed by the current study; therefore, further studies are needed to explore the mechanisms that cause a de- 
crease in stool calprotectin after red wine consumption. There was no change found in stool calprotectin levels in subjects without IBD, which is similar to other published reports [35].

We found that moderate daily consumption of red wine after only 1 week also disrupted intestinal barrier function in IBD patients. Small bowel permeability, as measured by urinary $\mathrm{L} / \mathrm{M}$ was increased significantly in patients with $\mathrm{CD}$ after moderate red wine consumption. However, the urinary $\mathrm{L} / \mathrm{M}$ ratio was not affected by red wine in patients with UC. In contrast, total gut (including the colon) permeability, as measured by urinary sucralose excretion, was increased in UC but not in CD patients after red wine consumption. These findings match the location of disease, as the $\mathrm{L} / \mathrm{M}$ reflects small bowel permeability and should therefore not be altered in UC. These findings suggest that moderate alcohol consumption disrupts intestinal permeability only at the areas of the gut that are already injured and thus more susceptible to the deleterious effects of alcohol. This notion is further supported by our findings that consumption of red wine did not significantly increase small bowel or colonic permeability in healthy subjects who had normal intestine prior to consumption of red wine.

Taken together, our findings suggest that consumption of a moderate amount of alcoholic beverages could potentially trigger IBD flare since prior studies have shown that increased intestinal permeability predict future flare-ups in IBD [15]. Moderate alcohol consumption has been associated with a number of health benefits in the majority of the population [36], but in the suscep- tible individual alcohol can have a number of deleterious effects including loss of intestinal permeability, endotoxemia, and immune activation. It is therefore not surprising that we found moderate short-term daily wine consumption to have seemingly contradictory effects on stool calprotectin and intestinal permeability in IBD patients. Thus, it is hard to speculate on the clinical consequences of moderate drinking on IBD disease course. Nonetheless, despite the immediate anti-inflammatory effects of alcohol as demonstrated by decreasing calprotectin, the long-term consequences of moderate alcohol consumption could be harmful because of the potential negative effects associated with gut leakiness on IBD disease course. Alcoholic beverages may therefore serve as a 'second hit' in susceptible individuals like patients with IBD and trigger activity of their disease.

We believe our findings are a 'proof of concept' that alcohol consumption can cause significant changes in markers of disease relapse in IBD. Limitations of the current study include its small sample size, limited duration of follow-up, and lack of assessment of mucosal activity. Our findings provide a rationale for future studies with larger sample size, longer follow-up, and endoscopic assessment to directly determine the long-term effects of moderate drinking on IBD disease course.

\section{Acknowledgements}

This study was supported in part by a research grant from Mrs. and Mr. Larry Field and by NIH grant AA13745.

\section{References}

1 Molodecky NA, Kaplan GG: Environmental risk factors for inflammatory bowel disease. Gastroenterol Hepatol (NY) 2010;6:339-346.

$\checkmark 2$ Cottone M, Rosselli M, Orlando A, et al: Smoking habits and recurrence in Crohn's disease. Gastroenterology 1994;106:643-648.

$\checkmark 3$ Cosnes J, Beaugerie L, Carbonnel F, Gendre JP: Smoking cessation and the course of Crohn's disease: an intervention study. Gastroenterology 2001;120:1093-1099.

4 Green JT, Rhodes J, Ragunath K, et al: Clinical status of ulcerative colitis in patients who smoke. Am J Gastroenterol 1998;93:14631467.

5 Aldhous MC, Drummond HE, Anderson N, et al: Smoking habit and load influence age at diagnosis and disease extent in ulcerative colitis. Am J Gastroenterol 2007;102:589597.

Moderate Red Wine Consumption and Inactive IBD
6 Patel M, Keshavarzian A, Kottapalli V, Badie B, Winship D, Fields JZ: Human neutrophil functions are inhibited in vitro by clinically relevant ethanol concentrations. Alcohol Clin Exp Res 1996;20:275-283.

7 Khoruts A, Stahnke L, McClain CJ, Logan G, Allen JI: Circulating tumor necrosis factor, interleukin-1 and interleukin- 6 concentrations in chronic alcoholic patients. Hepatology 1991;13:267-276.

$>8$ Bjarnason I, Peters TJ, Wise RJ: The leaky gut of alcoholism: possible route of entry for toxic compounds. Lancet 1984;1:179-182.

-9 Tang Y, Forsyth CB, Banan A, Fields JZ, Keshavarzian A: Oats supplementation prevents alcohol-induced gut leakiness in rats by preventing alcohol-induced oxidative tissue damage. J Pharmacol Exp Ther 2009;329: 952-958.
10 Mutlu E, Keshavarzian A, Engen P, Forsyth $\mathrm{CB}$, Sikaroodi M, Gillevet P: Intestinal dysbiosis: a possible mechanism of alcohol-induced endotoxemia and alcoholic steatohepatitis in rats. Alcohol Clin Exp Res 2009;33: 1836-1846.

11 Jowett SL, Seal CJ, Pearce MS, et al: Influence of dietary factors on the clinical course of ulcerative colitis: a prospective cohort study. Gut 2004;53:1479-1484.

12 Hey H, Schmedes A, Nielsen AA, Winding P, Gronbaek H: Effects of five different alcoholic drinks on patients with Crohn's disease. Scand J Gastroenterol 2007;42:968-972.

13 Swanson GR, Sedghi S, Farhadi A, Keshavarzian A: Pattern of alcohol consumption and its effect on gastrointestinal symptoms in inflammatory bowel disease. Alcohol 2010;44: 223-228.

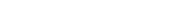


-14 Wyatt J, Oberhuber G, Pongratz S, et al: Increased gastric and intestinal permeability in patients with Crohn's disease. Am J Gastroenterol 1997;92:1891-1896.

15 Arnott ID, Kingstone K, Ghosh S: Abnormal intestinal permeability predicts relapse in inactive Crohn disease. Scand J Gastroenterol 2000;35:1163-1169.

16 Wild GE, Waschke KA, Bitton A, Thomson AB: The mechanisms of prednisone inhibition of inflammation in Crohn's disease involve changes in intestinal permeability, mucosal TNFalpha production and nuclear factor kappa B expression. Aliment Pharmacol Ther 2003;18:309-317.

-17 Gisbert JP, Bermejo F, Perez-Calle JL, et al: Fecal calprotectin and lactoferrin for the prediction of inflammatory bowel disease relapse. Inflamm Bowel Dis 2009;15:11901198.

- 18 Sandborn WJ, Feagan BG, Hanauer SB, et al: A review of activity indices and efficacy endpoints for clinical trials of medical therapy in adults with Crohn's disease. Gastroenterology 2002;122:512-530.

-19 Farhadi A, Keshavarzian A, Fields JZ, Sheikh M, Banan A: Resolution of common dietary sugars from probe sugars for test of intestinal permeability using capillary column gas chromatography. J Chromatogr B Analyt Technol Biomed Life Sci 2006;836:63-68.

-20 Farhadi A, Keshavarzian A, Holmes EW, Fields J, Zhang L, Banan A: Gas chromatographic method for detection of urinary sucralose: application to the assessment of intestinal permeability. J Chromatogr B Analyt Technol Biomed Life Sci 2003;784: 145-154.
21 Anderson AD, Poon P, Greenway GM, MacFie J: A simple method for the analysis of urinary sucralose for use in tests of intestinal permeability. Ann Clin Biochem 2005;42: 224-226.

22 Vermeire S, Van Assche G, Rutgeerts P: Laboratory markers in IBD: useful, magic, or unnecessary toys? Gut 2006;55:426-431.

23 Sakamoto N, Kono S, Wakai K, et al: Dietary risk factors for inflammatory bowel disease: a multicenter case-control study in Japan. Inflamm Bowel Dis 2005;11:154-163.

24 Mawdsley JE, Macey MG, Feakins RM, Langmead L, Rampton DS: The effect of acute psychologic stress on systemic and rectal mucosal measures of inflammation in ulcerative colitis. Gastroenterology 2006;131 410-419.

25 Keefer L, Stepanski EJ, Ranjbaran Z, Benson LM, Keshavarzian A: An initial report of sleep disturbance in inactive inflammatory bowel disease. J Clin Sleep Med 2006;2:409416.

26 Reid MC, Fiellin DA, O’Connor PG: Hazardous and harmful alcohol consumption in primary care. Arch Intern Med 1999;159: 1681-1689.

27 Adams PF, Schoenborn CA: Health behaviors of adults: United States, 2002-04. Vital Health Stat 10 2006;230:1-140.

28 Szabo G, Mandrekar P: A recent perspective on alcohol, immunity, and host defense. Alcohol Clin Exp Res 2009;33:220-232.
29 Bode C, Bode JC: Activation of the innate immune system and alcoholic liver disease: effects of ethanol per se or enhanced intestinal translocation of bacterial toxins induced by ethanol? Alcohol Clin Exp Res 2005;29: 166S-171S.

30 Lakatos PL, Kiss LS, Palatka K, et al: Serum lipopolysaccharide-binding protein and soluble CD14 are markers of disease activity in patients with Crohn's disease. Inflamm Bowel Dis 2011;17:767-777.

-31 McGuckin MA, Eri R, Simms LA, Florin TH, Radford-Smith G: Intestinal barrier dysfunction in inflammatory bowel diseases. Inflamm Bowel Dis 2009;15:100-113.

32 Costa F, Mumolo MG, Ceccarelli L, et al: Calprotectin is a stronger predictive marker of relapse in ulcerative colitis than in Crohn's disease. Gut 2005;54:364-368.

33 Shah VO, Ferguson JE, Hunsaker LA, Deck LM, Vander Jagt DL: Natural products inhibit LPS-induced activation of pro-inflammatory cytokines in peripheral blood mononuclear cells. Nat Prod Res 2010;24:11771188.

34 Yusuf N, Nasti TH, Meleth S, Elmets CA: Resveratrol enhances cell-mediated immune response to DMBA through TLR4 and prevents DMBA induced cutaneous carcinogenesis. Mol Carcinog 2009;48:713-723.

35 Montalto M, Gallo A, Ferrulli A, et al: Fecal calprotectin concentrations in alcoholic patients: a longitudinal study. Eur J Gastroenterol Hepatol 2011;23:76-80.

>36 O’Keefe JH, Bybee KA, Lavie CJ: Alcohol and cardiovascular health: the razor-sharp double-edged sword. J Am Coll Cardiol 2007;50: 1009-1014. 